\title{
Testosterone replacement in aging men: an evidence-based patient-centric perspective
}

\author{
Shalender Bhasin \\ Boston Claude D. Pepper Older Americans Independence Center and Research Program in Men's health: Aging and Metabolism, Brigham and Women's Hospital, Harvard Medical School, \\ Boston, Massachusetts, USA.
}

$T_{h}$ he deluge of advertisements marketing erectile dysfunction medications and testosterone products has empowered many older men to seek medical help for their sexual and genitourinary problems. As a reflection of this historical transition toward increased attention on men's sexual health, men's health clinics have sprung up across the United States; concomitantly, testosterone prescription sales increased from about $\$ 100$ million US dollars in the year 2000 to nearly $\$ 2.7$ billion in 2013.

Today, a majority of testosterone prescriptions are written for men aged 40-64 years (1) even though testosterone is not approved by the US Food and Drug Administration (FDA) for age-related decline in testosterone. Citing the lack of data on long-term benefits and risks of testosterone treatment in older men with agerelated decline, the FDA has sounded alarm over the growing off-label use of testosterone (2). Experts have debated whether prescribing testosterone to older men with testosterone deficiency is disease mongering or whether subsets of older men with testosterone deficiency might benefit from testosterone treatment. Fortunately, several recent randomized controlled trials (RCTs) have provided important information on the efficacy and short-term safety of testosterone treatment in older men (3-9). This Viewpoint synthesizes data from epidemiologic studies and RCTs and offers a perspective on a patient-centric approach to treatment decision in older men with testosterone deficiency based on an individualized assessment of benefits and risks.

\section{Epidemiological evidence}

Testosterone levels decline gradually with advancing age after peaking in the second and third decades of life $(10,11)$. Genetic factors, adiposity, and comorbid conditions affect the trajectory of age-related decline in testosterone levels $(10,12,13)$. Seven percent to $14 \%$ of community-dwelling middle-aged and older men have low morning fasting total testosterone below $250 \mathrm{ng} / \mathrm{dL}(10,11)$. The proportion of men who have low testosterone as well as sexual symptoms increases from $0.1 \%$ in men aged 40 to 49 years to $5.1 \%$ among those 70 to 79 years old (10). The majority of middle-aged and older men with low testosterone has low or inappropriately normal luteinizing hormone (LH) levels (secondary hypogonadism) and a smaller fraction has elevated LH (primary hypogonadism). Secondary hypogonadism is typically associated with obesity and comorbid conditions, while primary hypogonadism is more robustly associated with age (13).

Low testosterone levels in men are associated with low sexual desire and erectile dysfunction (ED); reduced skeletal muscle mass and strength, and impaired physical function; decreased bone mineral density (BMD); and increased risk of osteoporotic fractures. Measured and genetically determined estradiol levels in Mendelian randomization studies are more
Conflict of interest: SB reports receiving research grants from AbbVie, Transition Therapeutics, Metro International Biotechnology, and Alivegen. He has previously served as a member and chair of the American Board of Internal Medicine Endocrinology and Diabetes Board and chair of the Endocrine Society's expert panel that developed the guideline for testosterone treatment of hypogonadal men. He holds a patent (W02014138026A1) for an algorithm to calculate free testosterone concentrations, and stock holdings in Johnson and Johnson, CSL, Ltd (Australia), Abbott Laboratories, Thermo Fisher, and Danaher. He has served as a chair of the Data and Safety Monitoring Board for a clinical trial sponsored by OPKO, Inc.

Copyright: () 2021, American Society for Clinical Investigation.

Reference information: / Clin Invest. 2021;131(4):e146607. https://doi.org/10.1172/JCl146607. strongly associated with BMD and fracture risk than testosterone levels (14).

Low testosterone as well as sex hormone-binding globulin (SHBG) levels are each independently associated with increased risk of type 2 diabetes mellitus (T2DM) (12). In Mendelian randomization studies, higher testosterone level reduces the risk of $\mathrm{T} 2 \mathrm{DM}$ in men but increases the risk of T2DM in women (12). Low testosterone levels are associated with shorter telomere length and increased all-cause mortality $(15,16)$. Low testosterone level may be a marker of poor health.

\section{Placebo-controlled trials}

Among the small number of RCTs of the effects of testosterone in older men (3-9), the Testosterone Trials (the TTrials), a set of seven coordinated placebo-controlled trials, provided the most comprehensive evaluation of the efficacy of raising testosterone levels in older men (age 65 or older) who had an average of two-morning, fasting testosterone levels below $275 \mathrm{ng} /$ $\mathrm{dL}$ plus one or more conditions associated with low testosterone (low libido, mobility limitation, and fatigue). The TTrials and other RCTs found that testosterone treatment of men with low libido significantly improves overall sexual activity, sexual desire, and erectile function $(4,5$, 8). Testosterone treatment increases spontaneous sexual thoughts, attentiveness to erotic cues, duration and frequency of nocturnal penile erections, and ejaculate volume. Testosterone treatment does not improve sexual function in men with normal testosterone level who do not have sexual symptoms (6) or ejaculatory function in men with ejaculatory disorder and low testosterone level. In men with ED and low testosterone levels, treatment with phosphodiesterase 5 inhibitors is associated with substantial improvement in erectile function and the addition of testos- 


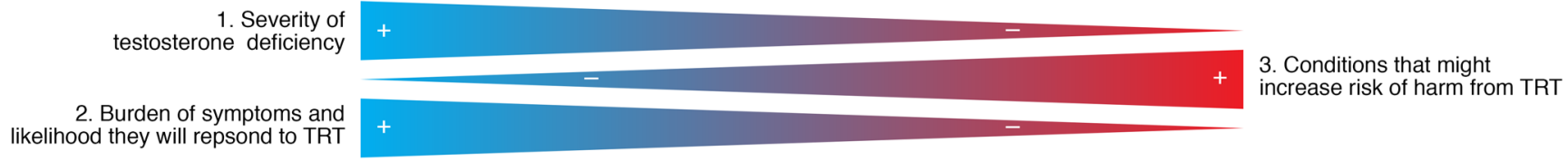

4. Weigh burden of symptoms and patient's values against potential risks/benefits

Potential benefits: Potential risks:

Strong evidence of efficacy Adverse events for which there is strong evidence

Improves sexual activity, sexual desire, and erections Erythrocytosis in men with low libido Acne

Corrects unexplained anemia in the elderly Detection of subclinical prostate cance

Suggestive evidence of efficacy Growth of metastatic prostate cancer

Improves depressive symptoms in patients with Reduced sperm production and fertility

late-life persistent depressive disorder (dysthymia)

Adverse events for which there is weak evidence

Gynecomastia

Modestly improves mobility in older adults with mobility limitation

Insufficient data or inconclusive evidence of efficacy

Improves fatigue

Reduces bone fracture risk

Evidence of lack of efficacy

Improves cognitive function in older men without cognitive deficit Improves major depressive disorder

Growth of balding (familia)

Growth of breast cancer

Induction of worsening of obstructive sleep apnea

Adverse events with insufficient data to assess risk

Major cardiovascular events

Prostate cancer

Treat $\longrightarrow$ Lean toward treatment $\longrightarrow$ Lean against treatment $\longrightarrow$ Do not

Figure 1. An evidence-based, individualized approach to testosterone therapy in older men with testosterone deficiency. The decision to offer testosterone treatment to older men with low testosterone levels should be guided by an individualized assessment of potential benefits and risks. Testosterone deficiency needs to be evaluated using reliable assays for the measurement of total and free testosterone levels. Patients should also be evaluated for conditions that are likely to respond to testosterone replacement therapy (TRT) as well as conditions that could be adversely impacted, such as prostate cancer, erythrocytosis, heart failure, or a hypercoagulable state. It is important to consider each patient's burden of symptoms, individual preferences, and risk tolerance against the uncertainty of long-term benefits and risks, the burden and risks of monitoring, and the cost.

terone does not further improve erectile function compared with placebo (17).

Testosterone treatment dose-dependently increases skeletal muscle mass, maximal voluntary muscle strength, and leg power, and modestly improves stair climbing power, 6-minute walking distance (6MWD), and self-reported mobility (18, 19). In the TTrials (4), testosterone-treated men were more likely to report that their walking ability had improved, suggesting that these modest improvements in 6MWD may be clinically meaningful. Testosterone administration also modestly improves aerobic capacity and attenuates the age-related decline in peak oxygen uptake $\left(\mathrm{VO}_{\text {2peak }}\right)$. Resistance exercise training augments the anabolic effects of androgens on muscle mass and strength.

Testosterone modestly improves depressive symptoms in hypogonadal men who do not have a depressive disorder $(4,20)$. In men with major depressive disorder (20), testosterone treatment, administered alone or along with antidepressant pharmacotherapy, has not been found to improve depression. Small RCTs suggest that testosterone therapy can improve depressive symptoms in men with late-life persistent depressive disorder (dysthymia) (20).
In young hypogonadal men, testosterone treatment improves areal as well as volumetric BMD, trabecular architecture, and estimated bone strength. In the TTrials, testosterone treatment of older hypogonadal men increased volumetric BMD and estimated bone strength in the hip and spine more than placebo (21). None of the testosterone trials has been sufficiently long or large to determine its effect on fracture risk. Testosterone might also reduce fall propensity because of its effects on muscle strength and reaction time.

The RCTs of the effects of testosterone on cognitive function are limited and equivocal. Some studies reported improvements in verbal memory and visuospatial skill while others found no effect. In general, testosterone treatment of older hypogonadal men without cognitive deficit has not improved cognitive function $(22,23)$. A few short-term studies in men with Alzheimer's disease $(\mathrm{AD})$ have reported modest improvements in verbal and spatial memory with testosterone treatment but small sample sizes, short intervention durations, and inclusion of men with normal testosterone and without confirmed AD limit their interpretability.

Testosterone administration reduces whole-body and visceral fat mass. Some trials have reported greater improvement in measures of insulin resistance with testosterone treatment of men with T2DM or metabolic syndrome than placebo (7), but testosterone treatment has not consistently improved glycemic control. The results of a large RCT in men with impaired glucose tolerance or T2DM (T4DM Trial) suggest that testosterone treatment administered in conjunction with a lifestyle program is more efficacious than the lifestyle program alone in reducing the proportion of men with T2DM (24).

Testosterone treatment of older men increases circulating erythrocytes, platelets, neutrophils, and monocytes. Testosterone treatment is more efficacious than placebo in correcting unexplained anemia of aging. Testosterone stimulates erythropoiesis by suppressing hepcidin transcription and increasing iron availability. Additionally, testosterone acts on hematopoietic progenitors to increase the numbers of myeloid progenitors, and stimulates erythropoietin. In spite of the increase in hemoglobin, 2,3-bisphosphoglycerate (BPG), muscle capillarity and blood flow, and mitochondrial biogenesis, testosterone has not been shown to improve fatigue (3). 


\section{Potential risks of testosterone treatment}

Testosterone treatment of older hypogonadal men is associated with a low frequency of adverse events (4-9). The adverse effects of testosterone treatment include acne, erythrocytosis, growth of metastatic prostate cancer, reduced sperm production, and increased risk of detecting subclinical prostate cancer (ref. 1 and Figure 1). Erythrocytosis is the most frequent adverse event associated with testosterone treatment. Testosterone treatment does not worsen lower urinary tract symptoms or obstructive sleep apnea. No adequately powered trial of sufficiently long duration has been conducted to determine the effects of testosterone on the risk of prostate cancer or major adverse cardiovascular events (MACE). Retrospective analyses of electronic medical records and meta-analyses of RCTs have yielded inconclusive results. No consistent relationship exists between testosterone levels and prostate cancer risk, and the rates of prostate cancer in the carefully selected men who were included in RCTs have been low. A Mendelian randomization analysis found that genetically determined testosterone level is associated with increased risk of prostate cancer (12); conversely, men with Klinefelter syndrome have decreased prostate cancer risk. These data suggest that long-term testosterone exposure could increase the risk of prostate cancer. An ongoing large cardiovascular safety RCT (TRAVERSE Trial, NCT03518034) in hypogonadal men, age 45-80 years, at increased cardiovascular risk, will provide definitive information on the effects of long-term testosterone treatment on MACE and other efficacy and safety outcomes.

\section{An individualized approach to treatment decision}

Testosterone treatment of older men with symptomatic testosterone deficiency offers some clinical benefits (e.g., improvement of sexual symptoms in men with low libido, correction of anemia) and is associated with low frequency of adverse events. However, because of the lack of evidence of long-term safety and limited evidence of long-term efficacy, testosterone treatment of all older men with low testosterone levels is not justified.
Instead, an expert panel of the Endocrine Society recommended that "testosterone therapy should be offered on an individualized basis ... in men $>65$ years who have symptoms or conditions suggestive of testosterone deficiency (e.g., low libido or unexplained anemia) and consistently low testosterone" (25).

The decision to offer testosterone treatment to older men with low testosterone levels should be guided by an individualized assessment of potential benefits and risks (Figure 1): (a) evaluate whether the patient has clear evidence of testosterone deficiency; (b) weigh the burden of symptoms/conditions against the potential benefits and the uncertainty of longterm harm; and (c) ascertain whether the patient has conditions, such as prostate cancer, erythrocytosis, heart failure, or a hypercoagulable state that would increase the risk of harm.

Older men considering testosterone treatment should undergo evaluation for prostate cancer risk. Prostate cancer screening and monitoring has some risks. The burden of symptoms, patient preferences, and risk tolerance should be weighed against the uncertainty of benefits and risks, the burden and risks of monitoring, and the cost. Finally, a shared decision to initiate testosterone treatment should be accompanied by a standardized monitoring plan to optimize the benefit-to-risk ratio.

Address correspondence to: Shalendar Bhasin, 221 Longwood Avenue, Room 545, Brigham and Women's Hospital, Boston, Massachusetts 02115, USA Phone: 617.525.9150; Email: sbhasin@ bwh.harvard.edu.

1. Nguyen CP, et al. Testosterone and "age-related hypogonadism"-FDA concerns. N Engl JMed. 2015;373(8):689-891.

2. Perls T, Handelsman DJ. Disease mongering of age-associated declines in testosterone and growth hormone levels. J Am Geriatr Soc. 2015;63(4):809-811.

3. Snyder PJ, et al. Effects of testosterone treatment in older men. N Engl JMed. 2016;374(7):611-624.

4. Brock $\mathrm{G}$, et al. Effect of testosterone solution $2 \%$ on testosterone concentration, sex drive and energy in hypogonadal men: results of a placebo controlled study. J Urol. 2016;195(3): 699-705.

5. Basaria S, et al. Effects of testosterone administration for 3 years on subclinical atherosclerosis progression in older men with low or low-normal testosterone levels: a randomized clinical trial.
JAMA. 2015;314(6):570-581.

6. Jones $\mathrm{TH}$, et al. Testosterone replacement in hypogonadal men with type 2 diabetes and/or metabolic syndrome (the TIMES2 study). Diabetes Care. 2011;34(4):828-837.

7. Steidle C, et al. AA2500 testosterone gel normalizes androgen levels in aging males with improvements in body composition and sexual function. J Clin Endocrinol Metab. 2003;88(6):2673-2681.

8. Travison TG, et al. Clinical meaningfulness of the changes in muscle performance and physical function associated with testosterone administration in older men with mobility limitation. J Gerontol A Biol Sci Med Sci. 2011;66(10):1090-1099.

9. Ponce OJ, et al. The efficacy and adverse events of testosterone replacement therapy in hypogonadal men: A systematic review and meta-analysis of randomized, placebo-controlled trials [published online March 17, 2018]. JClin Endocrinol Metab. https://doi.org/10.1210/jc.2018-00404.

10. Wu FC, et al. Identification of late-onset hypogonadism in middle-aged and elderly men. $N$ Engl J Med. 2010;363(2):123-135.

11. Bhasin S, et al. Reference ranges for testosterone in men generated using liquid chromatography tandem mass spectrometry in a communitybased sample of healthy nonobese young men in the Framingham Heart Study and applied to three geographically distinct cohorts. J Clin Endocrinol Metab. 2011;96(8):2430-2439.

12. Ruth KS, et al. Using human genetics to understand the disease impacts of testosterone in men and women. Nat Med. 2020;26(2):252-258.

13. Wu FC, et al. Hypothalamic-pituitary-testicular axis disruptions in older men are differentially linked to age and modifiable risk factors: the European Male Aging Study. J Clin Endocrinol Metab. 2008;93(7):2737-2745.

14. Nethander M, et al. Evidence of a causal effect of estradiol on fracture risk in men. JClin Endocrinol Metab. 2019;104(2):433-442.

15. Yeap BB, et al. In older men an optimal plasma testosterone is associated with reduced allcause mortality, and higher dihydrotestosterone with reduced ischaemic heart disease mortality, while estradiol levels do not predict mortality. J Clin Endocrinol Metab. 2014;99(1):E9-E18.

16. Araujo AB, et al. Clinical review: Endogenous testosterone mortality in men: a systematic review meta-analysis. JClin Endocrinol Metab. 2011;96(10):3007-3019.

17. Spitzer M, et al. Effect of testosterone replacement on response to sildenafil citrate in men with erectile dysfunction: a parallel, randomized trial. Ann Intern Med. 2012;157(10):681-691.

18 . Bhasin S, et al. Effect of testosterone replacement on measures of mobility in older men with mobility limitation and low testosterone concentrations: secondary analyses of the Testosterone Trials. Lancet Diabetes Endocrinol. 2018;6(11):879-890.

19. Storer TW, et al. Effects of testosterone supplementation for 3 years on muscle performance and physical function in older men. J Clin Endocrinol Metab. 2017;102(2):583-593.

20. Bhasin S, Seidman S. Testosterone treatment of depressive disorders in men: too much smoke, 
not enough high-quality evidence. JAMA Psychiatry. 2019;76(1):9-10.

21. Snyder PJ, et al. Effect of testosterone treatment on volumetric bone density and strength in older men with low testosterone: a controlled clinical trial. JAMA Intern Med. 2017;177(4):471-479.

22. Resnick SM, et al. Testosterone treatment and cognitive function in older men with low testosterone and age-associated memory impairment.
JAMA. 2017;317(7):717-727.

23. Huang $G$, et al. Effects of long-term testosterone administration on cognition in older men with low or low-to-normal testosterone concentrations: a prespecified secondary analysis of data from the randomised, double-blind, placebocontrolled TEAAM trial. Lancet Diabetes Endocrinol. 2016;4(8):657-665.

24. Wittert $\mathrm{G}$, et al. Testosterone treatment to pre- vent or revert type 2 diabetes in men enrolled in a lifestyle programme (T4DM): a randomised, double-blind, placebo-controlled, 2-year, phase $3 \mathrm{~b}$ trial. Lancet Diabetes Endocrinol. 2021;9(1):32-45.

25. Bhasin S, et al. Testosterone therapy in men with hypogonadism: an endocrine society clinical practice guideline. JClin Endocrinol Metab. 2018;103(5):1715-1744. 